\title{
Condrosarcoma tóraco-abdominal*
}

\author{
Dr. FELIPE FUENZALIDA S. ${ }^{1}$, Int. JUAN PABLO ESPINOZA G. ${ }^{2}$, Drs. ROBERTO CHARLES C. ${ }^{1}$, \\ GONZALO CARRASCO A. ${ }^{3}$, RICARDO ESPINOZA G. ${ }^{1}$
}

\begin{abstract}
Servicio de Cirugía, Hospital Parroquial de San Bernardo. Facultad de Medicina Universidad de los Andes.
Interno de Medicina, Facultad de Medicina Universidad de los Andes.

Servicio de Anatomía Patológica, Hospital Parroquial de San Bernardo.

Santiago, Chile.
\end{abstract}

\section{Abstract \\ Thoraco-abdominal chondrosarcoma}

Chondrosarcomas represent the third primary malignant bone tumor. Costal location occurs in $12 \%$, being the most common tumor of the ribs. It affects most frequently in the second and fifth decades of life. This tumor is histologically classified into grades 1,2 and 3 in descending order of differentiation. For grade 2 , with oncologic resection, there is a 10 -year survival of $64 \%$, and a $10 \%$ risk for metastasis. Radiotherapy has a limited therapeutic role and chemotherapy has not shown benefits. We report a case of a 45 years old woman, who presented with a symptomatic growing mass in the right rib cage, of three months of evolution, whose evaluation by magnetic resonance imaging and computed tomography shows a sarcomatous tumor with involvement of ribs, muscles, diaphragm, pleura, peritoneum and liver. A small amount of intraperitoneal free fluid was observed. A block resection was made, resulting a $16.8 \mathrm{~cm}$ long at the widest point chondrosarcoma, grade 2, with involvement of all layers of the wall, tumor microfoci in the liver capsule and malignant tumor cells in peritoneal fluid.

Key words: Chondrosarcoma, rib, histological grading.

\section{Resumen}

Los condrosarcomas representan la tercera neoplasia ósea maligna primaria. La ubicación costal representa el $12 \%$, siendo el tumor más frecuente de las costillas. Se presenta con mayor frecuencia en la segunda y quinta décadas de la vida. Este tumor se clasifica histológicamente en grados 1, 2 y 3 en orden decreciente de diferenciación. Para el grado 2, resecado oncológicamente, se observa una sobrevida a 10 años del 64\%; existiendo un $10 \%$ de riesgo de metástasis. La radioterapia tiene un escaso rol terapéutico y la quimioterapia no ha demostrado beneficios. Presentamos el caso de una mujer de 45 años que consultó por un aumento de volumen sintomático de la parrilla costal derecha, de tres meses de evolución, cuya evaluación a través de tomografía axial y resonancia magnética permitió delinear un tumor sarcomatoso de la pared con afectación de costillas, músculos, diafragma, pleura, peritoneo e hígado. Además se observaba escasa cantidad de líquido libre intraperitoneal. Fue resecado en block un condrosarcoma de $16,8 \mathrm{~cm}$ de eje mayor, grado 2, con compromiso de todos los planos de la pared, microfocos tumorales en cápsula hepática y células neoplásicas malignas en líquido peritoneal.

Palabra clave: Condrosarcoma, costilla, grado histológico.

*Recibido el 29 de marzo de 2011 y aceptado para publicación el 27 de julio de 2011.

Correspondencia: Dr. Ricardo Espinoza G.

San Carlos de Apoquindo 2200, Santiago, Chile. respinoza@uandes.cl 
Paciente de 45 años, sexo femenino, sin antecedentes mórbidos relevantes. Consulta por deformación de zona tóraco-abdominal anterior derecha, de unos 3 meses de evolución, progresiva, caracterizada por una elevación difusa de la parrilla costal. Es derivada a consulta quirúrgica tras la constatación de una masa en esa zona, acompañada con una tomografía axial que demostraba una tumoración de la pared costal de unos $15 \mathrm{~cm}$ en su eje mayor, con
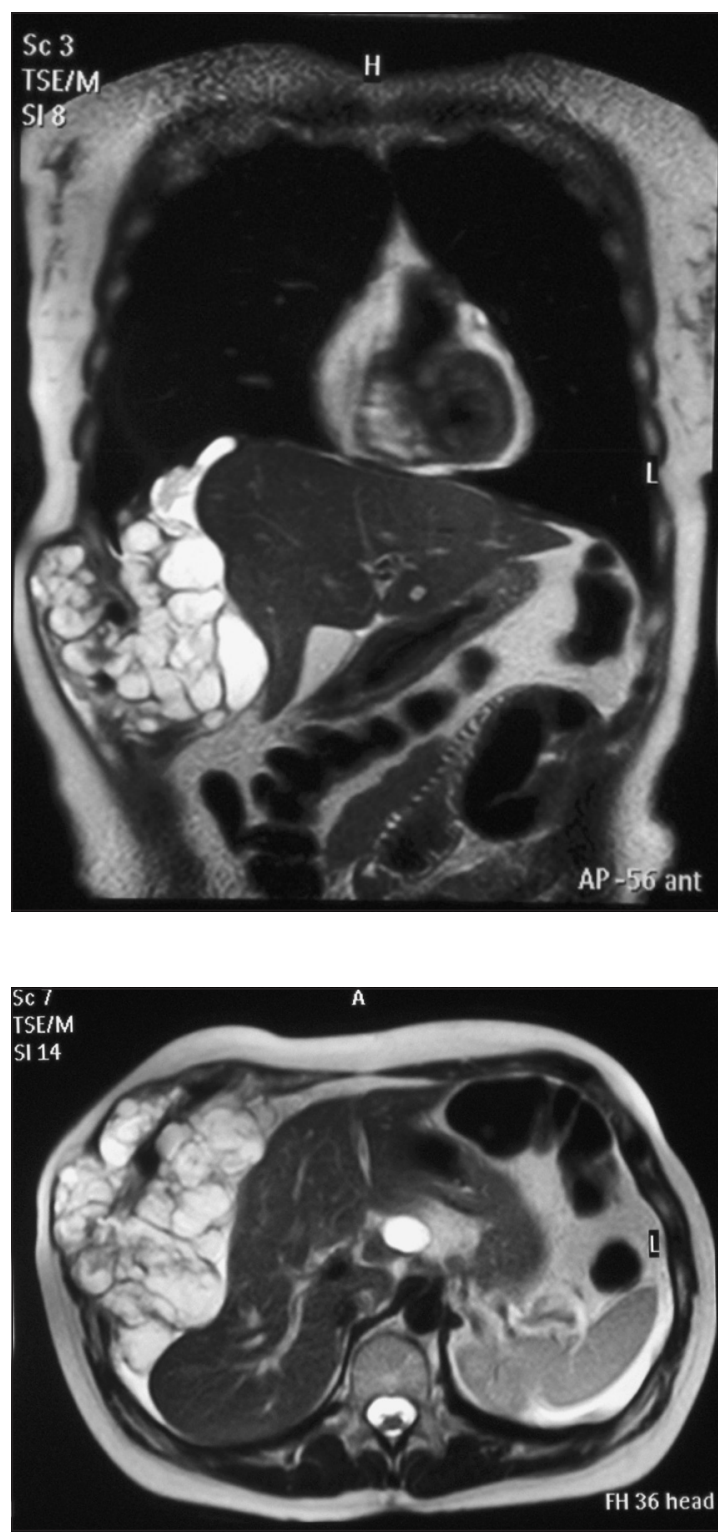

Figuras 1 y 2. Resonancia magnética que muestra lesión de la pared tóraco-abdominal que crece por sobre y bajo el diafragma derecho con compresión y deformación del hígado. probable compromiso pleural y peritoneal. Existía líquido libre en la cavidad peritoneal en pequeña cantidad. Se planteó la posibilidad de un sarcoma de partes blandas y se indicó completar estudio con resonancia magnética para una mejor definición de la afectación de los planos musculares, peritoneo e hígado. En este examen se confirmó la masa, el compromiso del diafragma y la probable infiltración de la pleura y el peritoneo sin pronunciamiento sobre su naturaleza. Se efectuó una biopsia incisional que demostró una tumoración constituida por un tejido denso, en parte gelatinoso, compatible con un condrosarcoma.

Se planificó cirugía oncológica resectiva, con intención curativa, extirpando en block la masa en la zona tóraco-abdominal comprometida, que incluyó cuatro costillas y prácticamente todo el hemidiafragma derecho con superficie pleural y peritoneal. Se envió líquido peritoneal a estudio citológico y se biopsió cápsula hepática.

Se reconstruyó el diafragma con una extensa malla de polipropileno, al igual que la pared costal. El postoperatorio cursó sin complicaciones y fue dada de alta una semana más tarde.

El informe anátomo-patológico diferido confirmó la existencia de un cáncer de la parrilla costal de $16,8 \mathrm{~cm}$ de eje mayor, tipo histológico condrosarcoma, grado histológico 2 (escala 1 al 3), extensamente infiltrante de costillas, músculos esqueléticos, tejido subcutáneo, diafragma, pleura, peritoneo y con microfocos tumorales en cápsula hepática. En el líquido peritoneal también se demostró presencia de células neoplásicas malignas.

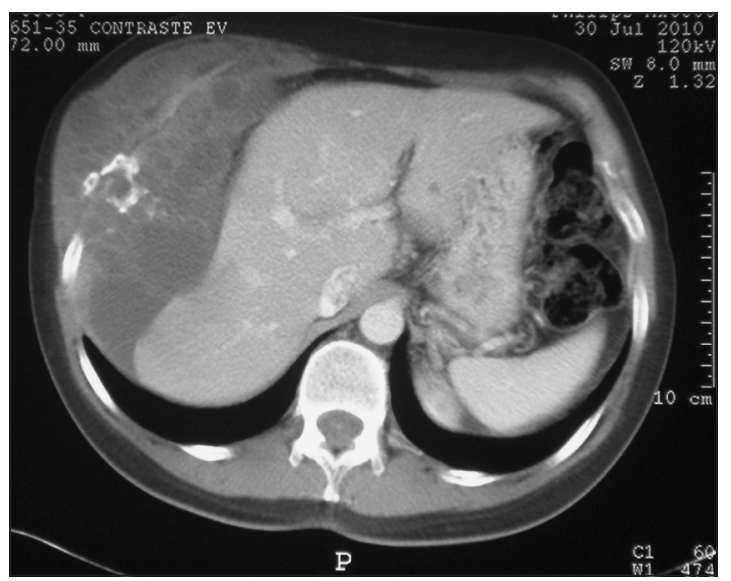

Figura 3. Tomografía computada muestra lesión tumoral de la pared de $15 \mathrm{~cm}$ en su eje mayor, con destrucción ósea. 


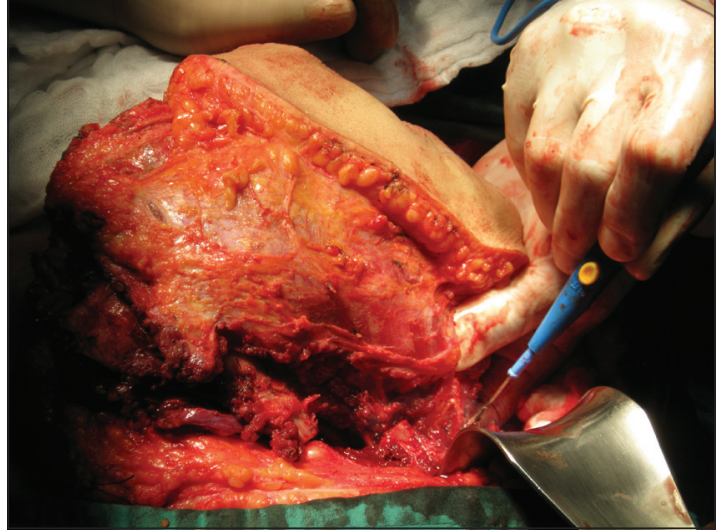

Figuras 4 y 5. Resección tumoral durante la cirugía.

\section{Discusión}

Los condrosarcomas son tumores malignos del hueso que se caracterizan por producir matriz cartilaginosa ${ }^{1}$. Representan la tercera neoplasia ósea maligna primaria ${ }^{2}$ y el principal de este tipo en la pared torácica ${ }^{3,4}$. El $90 \%$ se origina de novo, teniendo el resto como lesión precursora el osteocondroma y el osteosarcoma. La ubicación costal constituye un $12 \%$, siendo el tumor más frecuente de las costillas ${ }^{5}$. Suelen ocurrir en la unión costocondral y pared anterior. Se presentan en dos momentos de la vida con mayor frecuencia: segunda y quinta década, y con leve predominancia por el sexo masculino. La presentación más frecuente es masa palpable $(80 \%)$, aunque algunos pacientes pueden consultar por do$\operatorname{lor}^{3,4}$, síntoma que es más frecuente en los tumores de alto grado ${ }^{6}$.

La diferenciación histológica es uno de los factores pronósticos más importantes ${ }^{7}$. El tipo histológico más frecuente es el condrosarcoma convencional o central, constituyendo entre el $75 \%$ y un $90 \%$ de los $\operatorname{casos}^{8,9}$. Se clasifican en grado 1, 2 y 3 según tamaño nuclear, patrón de tinción, actividad mitótica y grado de celularidad, siendo el grado 1 el mejor diferenciado y de mejor pronóstico; aproximadamente el $90 \%$ de los pacientes se presentan con grado 1 ó $2^{10}$. La sobrevida a 10 años del tumor grado 2, resecado oncológicamente, es de aproximadamente $64 \%$. Este tiene hasta un $10 \%$ de riesgo de metástasis ${ }^{10,11}$.

La imagen radiológica más propia corresponde a una masa de la pared torácica con destrucción ósea y compromiso de partes blandas. Se pueden apreciar áreas de calcificación en la matriz cartilaginosa, especialmente en la tomografía computada ${ }^{4}$.

En la RNM se observan con señal intermedia en

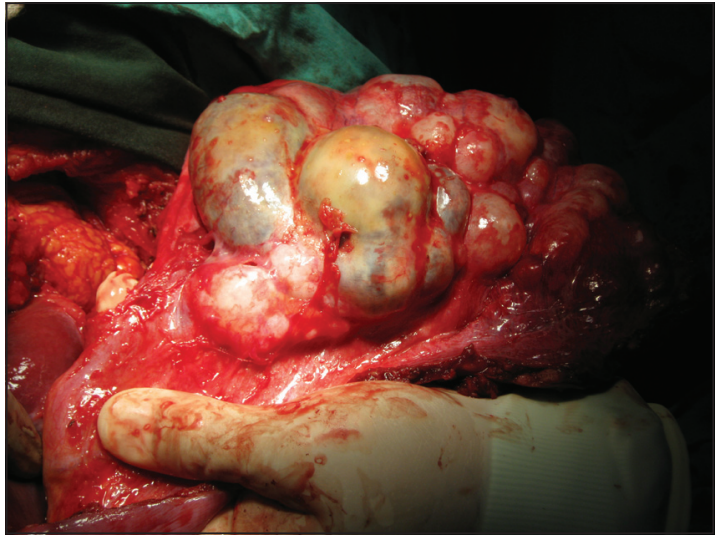

T1 y señal heterogénea en T2, con áreas de aumento de intensidad ${ }^{4,12}$.

El principal diagnóstico diferencial debe hacerse con un condroma; sin embargo, el compromiso cortical obliga a plantear un osteosarcoma, un fibrosarcoma, un plasmocitoma y, eventualmente, hasta una metástasis. Dependiendo de la localización, en un hueso largo también puede plantearse la posibilidad de un fibroma condromixoide, un condroblastoma o un tumor de células gigantes; en estos dos últimos casos si es de ubicación epifisiaria ${ }^{13}$.

El rol de la tomografía por emisión de positrones (PET) es prometedor pero incierto ${ }^{14}$.

Ante la sospecha de un condrosarcoma, se puede realizar biopsia incisional, buscando las zonas de mayor sospecha en las imágenes para mejorar el rendimiento ${ }^{15}$.

La etapificación de estos tumores se puede realizar mediante el sistema TNM planteado por la American Joint Committee on Cancer (AJCC); sin embargo, el sistema más frecuentemente utilizado corresponde al desarrollado por Enneking et al, que considera el grado tumoral, localización anatómica y presencia o no de metástasis ${ }^{16-18}$.

La única opción terapéutica curativa para lesiones no metastásicas es la resección completa con márgenes negativos ${ }^{3,4,19}$. La radioterapia tendría algún rol en el control de enfermedad residual en casos de resección incompleta, aunque se considera que los condrosarcomas son relativamente radiorresistentes por su bajo potencial mitótico ${ }^{20}$. La quimioterapia no ha demostrado beneficios ${ }^{21}$.

El pronóstico de una lesión resecada varía, por tanto, de acuerdo con los márgenes; en casos resecados con criterios R0 la sobrevida a 10 años es de hasta un $90 \%$ versus el $47 \%$ en caso de una resección incompleta. La tasa de recurrencia local es de $4 \%$ y $73 \%$ respectivamente ${ }^{3}$. 


\section{Referencias}

1. Fletcher CD, Unni KK, Mertens F. Cartilage tumours. En: Tumours of soft tissue and bone. Pathology and genetics. World Health Organization: classification of tumours. 2002:233.

2. Jemal A, Siegel R, Ward E, Murray T, Xu J, Smigal C, et al. Cancer statistics. Cancer J Clin. 2006;56:106-30.

3. Kim J, Hofstetter WL. Tumors of the mediastinum and chest wall. Surg Clin N Am. 2010;90:1019-40.

4. Gladish G, Sabloff B, Munden R, Truong M, Erasmus J, Chasen M. Primary thoracic sarcomas. Radio Graphics 2002;22:621-37.

5. Ehara S, Nakayama T, Nishida J, Shiraishi H, Yoshiaka $\mathrm{H}$, Aoki J. Bone scintigraphic an CT evaluation of chondrosarcoma of the rib: Correlation with histological grade in 6 cases. Ann Nucl Med. 2004;18:633-6.

6. Bashir SI, Gupta R, Khan HN, Ahmed R, Mohd A, Salaria AQ. Trans-articular chondrosarcoma grade 2 of proximal phalanx resulting in its fracture along with destruction of middle phalanx of $2^{\text {nd }}$ toe right foot: a case report and review of the literature. Cases J. 2009;2:7488.

7. Giuffrida AY, Burgueno JE, Koniaris LG, Gutiérrez JC, Duncan R, Scully SP. Chondrosarcoma in the United States (1973 to 2003): an analysis of 2890 cases from the SEER database. J Bone Joint Surg Am. 2009;91:1063-72.

8. Martínez F, Manjón P, Montes S. Condrosarcoma. Variantes de condrosarcoma. Rev Esp Patol. 2006;39:69-70.

9. Brien EW, Mirra JM, Kerr R. Benign and malignant cartilage tumors of bone and joint: their anatomic and theoretical basis with an emphasis on radiology, pathology and clinical biology. I. The intramedullary cartilage tumors. Skeletal Radiol. 1997;26:325-53.

10. Björnsson J, McLeod RA, Unni KK, Ilstrup DM, Prit- chard DJ. Primary chondrosarcoma of long bones and limb girdles. Cancer 1998;83:2105-19.

11. Evans HL, Ayala AG, Romsdahl MM. Prognostic factors in chondrosarcoma of bone: a clinicopathologic analysis with emphasis on histologic grading. Cancer 1977;40:818-31.

12. Murphey MD, Walker EA, Wilson AJ. From the archives of the AFIP: imaging of primary chondrosarcoma: radiologic-pathologic correlation. Radiographics 2003; 23:1245-78.

13. Ollivier L, Vanel D, Leclère J. Imaging of chondrosarcomas. Review. Cancer Imaging 2004;4:36-8.

14. Nanni C, Fanti S.FDG-PET and PET/CT for evaluating soft tissue sarcomas. PET Clin. 2010;5:341-7.

15. Normand AN, Cannon CP, Lewis VO, Lin PP, Yasko AW. Curettage of biopsy-diagnosed grade 1 periacetabularchondrosarcoma. Clin Orthop Relat Res. 2007;459:146-9.

16. Edge SB, Byrd DR, Compton CC. AJCC (American Joint Committee on Cancer) Cancer Staging Manual. 7th edition. New York: Springer;2010:281.

17. Enneking WF. A system of staging musculoskeletal neoplasms. Clin Orthop Relat Res. 1986;204:9-24.

18. Wolf RE, Enneking WF. The staging and surgery of musculoskeletal neoplasms. Orthop Clin North Am. 1996;27:473-81.

19. Fiorenza F, Abudu A, Grimer RJ, Carter SR, Tillman RM, Ayoub K, et al. Risk factors for survival and local control in chondrosarcoma of bone. J Bone Joint Surg Br. 2002;84:93-9.

20. Normand AN, Ballo MT, Yasko AW. Palliative radiation therapy for chondrosarcoma (abstract). Proc Connect Tissue Oncol Soc. 2006;12:745a.

21. Riedel RF, Larrier N, Dodd L. The clinical management of chondrosarcoma. Curr Treat Options Oncol. 2009;10:94-106. 